\title{
Processes of anaerobic oxidation of oil in bottom sediments of Lake Baikal
}

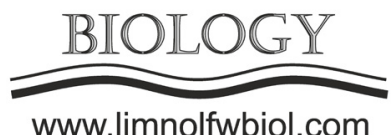

www.limnolfwbiol.com

\author{
Pavlova O.N.*, Izosimova O.N., Chernitsyna S.M., Ivanov V.G., Pogodaeva T.V., \\ Gorchkov A.G.
}

Limnological Institute, Siberian Branch of the Russian Academy of Sciences, Ulan-Batorskaya Str., 3, Irkutsk, 664033, Russia

\begin{abstract}
In the conditions of laboratory modelling, we studied the ability of microbial communities from the subsurface and deep sediments near the Gorevoy Utes oil seepage site to carry out the process of anaerobic oxidation of petroleum hydrocarbons. In the sudsurface sediments, the conversion of $n$-alkanes was the most intensive in the cultures enriched with sulfate ions, and in the deep ones - with bicarbonate ions. We determined a high biodegradation degree of polycyclic aromatic hydrocarbons (PAHs) in the deep sediment samples regardless of the electron acceptors that were present in the medium. Analysis of the 16S rRNA gene clone libraries of bacterial and archaeal communities revealed that libraries of the four investigated samples consist of the sequences that are $97-99 \%$ homologous to the uncultured microorganisms from the thermophilic habitats, mud volcano sediments and hydrocarbon polluted environments.
\end{abstract}

Keywords: anaerobic oxidation of petroleum hydrocarbons, microbial communities, bottom sediments, Lake Baikal

The processes of anaerobic oil oxidation in bottom sediments of freshwater lakes have not been previously studied. At Lake Baikal, there are two known sites with natural oil seepages. One site is located at the estuary of the Bolshaya Zelenovskaya River (BZ) and has been known since the late 18th century, another site is located near Cape Gorevoy Utes (GU) (discovered in 2005). The oil collected during its release to the water surface at the GU site in 2005 was characterized by an extremely high content of $n$-alkanes and was identified as nonbiodegradable paraffin oil (Kontorovich et al., 2007). At present, chemical analysis of the oil composition has indicated its biodegradation (Gorshkov et al., 2020). This work aimed to study the processes of anaerobic oxidation of oil by the microbial community from the subsurface and deep sediments of the Gorevoy Utes natural oil seep.

Samples of gas-hydrate and oil-bearing bottom sediments collected in 2018 from the Cape Gorevoy Utes (St. 5, GC-3) were investigated. To obtain enrichment cultures, we used sedimentary layers that corresponded to the core depths of $10-30 \mathrm{~cm}$ (GUI) and $100-120 \mathrm{~cm}$ (GUII). Cultivation was carried out at a temperature of $10^{\circ} \mathrm{C}$ in a mineral medium containing oil and an $\mathrm{N}_{2} / \mathrm{CO}_{2}$ gas mixture $(90: 10 \mathrm{v} / \mathrm{v})$. Sulfate-reducing conditions were determined in microcosms with the addition of $\mathrm{Na}_{2} \mathrm{SO}_{4}$ (final concentration of $20 \mathrm{mM}$ ) to the medium, and $\mathrm{Na}_{2} \mathrm{~S}(0.5 \mathrm{mM})$ was added as a reducing agent. Methanogenic microcosms were prepared as for sulfate- reducing microcosms but without the addition of $\mathrm{Na}_{2} \mathrm{SO}_{4}$. Hydrocarbon gases in experimental vials were determined by a modified phase-equilibrium degassing method (Mizandrontsev et al., 2020). The degree of oil utilization by the microbial community was inferred from the loss of $n$-alkanes and PAHs determined by gas chromatography-mass spectrometry (Gorshkov et al., 2020) after one year of cultivation. To analyse clone libraries of the enrichment cultures, 30 clones with fragments of the 16S rRNA gene sequences were taken from each sample. The Sanger reaction with the use of BigDye Terminator Kit v. 3.1 (Applied Biosystems, United States) and analysis of the reaction products on an ABI 3130x1 gene analyzer were carried out at the SB RAS Genomics Core Facility, Novosibirsk.

In the GUI sample, the highest loss of $n$-alkanes (28\%) was determined during the cultivation of the microbial community under sulfate-reducing conditions. Under methanogenic conditions, a decrease in the amount of alkane oil fraction was insignificant and equal to $6 \%$. In deep sedimentary layers, we observed the opposite pattern. In the cultures enriched with bicarbonate ion, the conversion of $n$-alkanes was $20 \%$, and those enriched with sulfate ion - less than $1.5 \%$. In the GUII, we recorded the highest conversion of PAHs (45-46\%) under methanogenic and sulfate-reducing conditions, respectively. In the subsurface sedimentary layer, the loss of the total PAHs was lower than in the deep one. In the presence of sulfate in the enrichment

*Corresponding author.

E-mail address: pavlova@lin.irk.ru (O.N. Pavlova)

(C) Author(s) 2020. This work is distributed under the Creative Commons Attribution 4.0 License. 
cultures, the $\Sigma_{\mathrm{PAH}}$ content decreased by $20 \%$, and in the presence of bicarbonate - by $37 \%$. We identified a significant formation of methane in the enrichment cultures of GUI. Its highest concentration $(32.5 \mathrm{mM} / \mathrm{l})$ was recorded after six months of cultivation. In the enrichment cultures of GUII, the concentration of the formed methane did not exceed $3.8 \mathrm{mM} / 1$. The loss of sulfate ions in the enrichment cultures containing both the subsurface and deep samples was $25 \%$ of the initial concentration.

Analysis of the 16S rRNA gene clone libraries of bacterial and archaeal communities revealed that libraries of all studied samples consisted of the sequences that were $97-99 \%$ homologous to the uncultured microorganisms from thermophilic habitats, mud volcano sediments and hydrocarbon polluted environments. In all 16S rRNA gene clone libraries of bacteria, we identified the members of the phyla Firmicutes (17-67\% of the total number of the analysed clones), Chloroflexi (7-37\%) and Proteobacteria $(\delta / \varepsilon)(3-$ $17 \%)$. We detected the representatives of the phylum Caldiserica (OP5) in three of the four gene clone libraries where their representativeness was from 30 to $43 \%$. The sequences of the phyla Acidobacteria, Bacteroidetes, Planctomycetes, Proteobacteria $(\alpha)$, Thermotogae, Ca. Aminicinantes (OP8), Ca. Atribacteria (OP9), Ca. Armatimonadetes (OP10), Ca. division (OP11), and Ca. division AC1 were minor. In three of the four 16S rRNA gene libraries of archaea (GUI_SO ${ }_{4}, \mathrm{GUII}_{-} \mathrm{CH}_{4}$, GUII_SO ${ }_{4}$ ), we determined a homogenous composition of the components represented by the sequences of the class Methanomicrobia (29 clones). In the gene library of the GUI_CH $\mathrm{CH}_{4}$ enrichment culture, in addition to the members of the phylum Euryarchaeota (classes Methanomicrobia, Methanococci, Thermoplasmata, and Thermococci), we detected the members of the clade of TACK group (Crenarchaeota, Bathyarchaeota and Aigarchaeota). Among the 16S rRNA gene libraries, the GUII_SO ${ }_{4}$ enrichment culture was the most diverse in the composition of components. The uncultured members of the phylum Caldiserica were numerically dominant in this culture, which was originally detected in Yellowstone hot springs as the 16S rRNA-based lineage 'OP5' (Hugenholtz et al., 1998). The members of the phyla Firmicutes and Chloroflexi were the second in distibution; they were 93-95\% homologous to the unclassified Thermoactinomycetaceae bacterium and Dehalococcoidia bacterium, respectively.

In the subsurface and deep sediments of the GU oil seep, through incubation of native samples under anaerobic conditions, we recorded the loss of $n$-alkanes and PAHs accompanied by the methane formation. In the subsurface sediments, the conversion of $n$-alkanes was the most intensive in the cultures enriched with sulfate ions; in the deep ones - with bicarbonate ions. In general, the microbial community of the deep sediments was more focused on the anaerobic oxidation of PAHs, as evidenced by their high biodegradation degree regardless of the electron acceptors that were present in the medium. The detection in all investigated samples of the sequences of the members of the bacterial phyla Proteobacteria $(\delta / \varepsilon)$ and Firmicutes as well as archaea of the class Methanomicrobia may also indicate syntrophic processes of oil degradation. The presence of numerous sequences of the thermophilic Candidate division "OP" (OP 5, 8, 9, 10, 11), Firmicutes and Thermoplasmata in the bacterial and archaeal 16S rRNA gene clone libraries may result from the entry of thermophilic prokaryotes together with gas-saturated fluids from the hydrocarbon generation zone of Lake Baikal.

\section{Acknowledgements}

This work was conducted within the framework of the State Task (project No. 0345-2019-0007 (AAAA-A16-116122110064-7) and RFBR grant no.16-04-00181. Cultivation was carried out in the dark at $10^{\circ} \mathrm{C}$ using the facilities of the Experimental Freshwater Aquarium Complex of Baikal Hydrobionts; Chromatography-mass-spectrometry was conducted at the Collective Instrumental Center "Ultramicroanalysis" (Limnological Institute SB RAS).

\section{References}

Gorshkov A., Pavlova O., Khlystov O. et al. 2020. Fractioning of petroleum hydrocarbons from seeped oil as a factor of purity preservation of water in Lake Baikal (Russia). Journal of Great Lakes Research 46: 115-122. DOI: 10.1016/j. jglr.2019.10.010

Hugenholtz P., Pitulle C., Hershberger K.L. et al. 1998. Novel division level bacterial diversity in a Yellowstone hot spring. Journal of Bacteriology 180: 366-376. DOI: 10.1128/ jb.180.2.366-376.1998

Mizandrontsev I.B., Kozlov V.V., Ivanov V.G. et al. 2020. Vertical distribution of methane in Baikal water. Water resources 47: 122-129. DOI: 10.1134/s0097807820010108

Kontorovich A.E., Kashirtsev V.A., Moskvin V.I. 2007. Petroleum potential of Baikal deposits. Russian Geology and Geophysics 48: 1046-1053. DOI: 10.1016/j.rgg.2007.11.004 